\title{
LINEAR OPERATORS THAT PRESERVE SOME TEST FUNCTIONS
}

\author{
OCTAVIAN AGRATINI
}

Received 6 October 2005; Revised 23 March 2006; Accepted 4 April 2006

The paper centers around a pair of sequences of linear positive operators. The former has the degree of exactness one and the latter has its degree of exactness null, but, as a novelty, it reproduces the third test function of Korovkin theorem. Quantitative estimates of the rate of convergence are given in different function spaces traveling from classical approximation to approximation in abstract spaces. Particular classes are also studied.

Copyright @ 2006 Hindawi Publishing Corporation. All rights reserved.

\section{Introduction}

One of the most powerful and spectacular criteria to help us decide if a sequence $\left(L_{n}\right)_{n \geq 1}$ of positive linear operators tends to the identity operator with respect to the uniform norm of the space $C([a, b])$ was established by P. P. Korovkin and H. Bohman in the fifties. This theorem says: if $\left(L_{n} e_{k}\right)_{n \geq 1}$ converges to $e_{k}$ uniformly on $[a, b], k \in\{0,1,2\}$, for the test functions $e_{0}(x)=1, e_{1}(x)=x, e_{2}(x)=x^{2}$, then $\left(L_{n} f\right)_{n \geq 1}$ converges to $f$ uniformly on $[a, b]$, for each $f \in C([a, b])$.

Many classical linear positive operators preserve $e_{0}$. For example, all operators which can be defined by using probabilistic methods enjoy this property. Besides, a part of these operators also preserves the second test function $e_{1}$, this implying that the affine functions are invariant by them. It is known that if a linear positive operator preserved all three test functions, it would then become the identity operator, so it is not useful in approximation theory.

Recently, King [8] has presented an unexpected example of operators of Bernstein type which preserve $e_{0}$ and $e_{2}$. Motivated by this work, in the present paper which comprises five Sections, we focus our attention on indicating a general technique to construct sequences of operators of discrete type with the same property as in King's example. By using different moduli of smoothness, we study the rate of local and global estimates for our class in various functions spaces. A probabilistic approach is also mentioned. Particular cases are analyzed and, following our technique, we give the modified variants of Szász, Baskakov, and Bernstein-Chlodovsky operators. The last section is devoted to 


\section{Linear operators that preserve some test functions}

identifying what this property would mean in some abstract spaces and to presenting a new look of the rate of convergence for the operators which satisfy it.

\section{Construction of the class $\left(L_{n}^{*}\right)_{n \geq 1}$}

Throughout the paper, $\mathbb{R}_{+}$stands for the interval $[0, \infty)$ and $\mathbb{N}_{0}$ denotes the set $\{0\} \cup \mathbb{N}$. At first step, we consider a sequence $\left(L_{n}\right)_{n \geq 1}$ of linear positive operators of discrete type acting on a subspace of $C\left(\mathbb{R}_{+}\right)$and defined as follows:

$$
\left(L_{n} f\right)(x)=\sum_{k=0}^{\infty} u_{n, k}(x) f\left(x_{n, k}\right), \quad x \geq 0, f \in \mathscr{F} \cap E_{\alpha},
$$

where every function $u_{n, k}: \mathbb{R}_{+} \rightarrow \mathbb{R}_{+}$is continuous $\left(n \in \mathbb{N}, k \in \mathbb{N}_{0}\right),\left(x_{n, k}\right)_{k \geq 0}:=\Delta_{n}$ is a net on $\mathbb{R}_{+}$, and

$$
\begin{gathered}
\mathscr{F}:=\left\{f \in \mathbb{R}_{+}^{\mathbb{R}_{+}}: \text {the series in (2.1) is convergent }\right\}, \\
E_{\alpha}:=\left\{f \in C\left(\mathbb{R}_{+}\right): \frac{f(x)}{1+x^{\alpha}} \text { is convergent as } x \longrightarrow \infty\right\},
\end{gathered}
$$

$\alpha \geq 2$ being fixed. The space $E_{\alpha}$ is endowed with the usual norm $\|\cdot\|_{\alpha}^{*},\|f\|_{\alpha}^{*}:=$ $\sup _{x \geq 0}\left(|f(x)| /\left(1+x^{\alpha}\right)\right)$. Clearly, if $\alpha<\alpha^{\prime}$, then $E_{\alpha} \subset E_{\alpha^{\prime}}$. We mention that the right-hand side of (2.1) could be a finite sum. For example, if we take $u_{n, k}(x)=\left(\begin{array}{l}n \\ k\end{array}\right)(1+x)^{-n} x^{k}$ for $k=0,1, \ldots, n, u_{n, k}=0$ for $k>n$, and we choose the net $\Delta_{n}=(k /(n-k+1))_{k=\overline{0, n}}$, then $L_{n}$, $n \in \mathbb{N}$, become the well-known Bleimann-Butzer-Hahn operators [3].

In order to obtain for $L_{n}$ the degree of exactness one, we consider the following identities:

$$
\sum_{k=0}^{\infty} u_{n, k}(x)=1, \quad \sum_{k=0}^{\infty} u_{n, k}(x) x_{n, k}=x, \quad x \geq 0,
$$

are fulfilled for each $n \in \mathbb{N}$. We also impose that $L_{n}$ maps $e_{2}$ in a polynomial of second degree, more precisely,

$$
\left(L_{n} e_{2}\right)(x)=a_{n} x^{2}+b_{n} x+c_{n}, \quad x \geq 0,
$$

where $a_{n} \neq 0, n \in \mathbb{N}, \lim _{n \rightarrow \infty} a_{n}=1, \lim _{n \rightarrow \infty} b_{n}=\lim _{n \rightarrow \infty} c_{n}=0$.

Based on Bohman-Korovkin theorem, the values of these limits and relation (2.3) guarantee that $\left(L_{n}\right)_{n \geq 1}$ is an approximation process on any compact $K \subset \mathbb{R}_{+}$. Clearly, $\left\{e_{0}, e_{1}, e_{2}\right\} \subset E_{\alpha}$. Relations (2.3) and (2.4) guarantee that $\left\{e_{0}, e_{1}, e_{2}\right\} \subset \mathscr{F}$. Resulted from Cauchy-Schwarz inequality,

$$
\left(L e_{1}\right)^{2} \leq\left(L e_{0}\right)\left(L e_{2}\right)
$$

is a common property of any linear positive operator $L$ of summation type. Based on it, we get

$$
\left(a_{n}-1\right) x^{2}+b_{n} x+c_{n} \geq 0, \quad x \geq 0, n \in \mathbb{N},
$$


which implies

$$
c_{n} \geq 0, \quad a_{n} \geq 1 \quad \text { for each } n \in \mathbb{N} \text {. }
$$

Moreover, $\left\{n \in \mathbb{N}: a_{n}=1\right\} \subset\left\{n \in \mathbb{N}: b_{n} \geq 0\right\}$.

At this moment, we are looking for the functions $v_{n} \in \mathbb{R}_{+}^{\mathbb{R}_{+}}, n \in \mathbb{N}$, such that $\left(L_{n} e_{2}\right)$ $\left(v_{n}(x)\right)=x^{2}$ for each $x \geq 0$ and each $n \in \mathbb{N}$, this means

$$
a_{n} v_{n}^{2}(x)+b_{n} v_{n}(x)+c_{n}-x^{2}=0, \quad x \geq 0, n \in \mathbb{N} .
$$

Lemma 2.1. Let $L_{n}, n \in \mathbb{N}$, be defined by (2.1) such that (2.3) and (2.4) hold. The functions $v_{n}, n \in \mathbb{N}$, given by (2.8) exist, if and only if the following conditions take place:

$$
\begin{gathered}
b_{n}^{2} \geq 4 a_{n} c_{n}, \quad n \in \mathbb{N}, \\
\left\{n \in \mathbb{N}: c_{n} \neq 0\right\} \subset\left\{n \in \mathbb{N}: b_{n} \leq 0\right\} .
\end{gathered}
$$

One has

$$
v_{n}(x)=\frac{1}{2 a_{n}}\left(\sqrt{b_{n}^{2}+4 a_{n}\left(x^{2}-c_{n}\right)}-b_{n}\right), \quad x \geq 0 .
$$

Proof. Let $n \in \mathbb{N}$ be arbitrarily fixed. Suppose that (2.8) holds. Since $v_{n}(0)$ must be a real number, we get $b_{n}^{2} \geq 4 a_{n} c_{n}$. If $c_{n}=0,(2.8)$, see also relation (2.7), permits us to find a nonnegative $v_{n}(x)$ for each $x \geq 0$. If $c_{n} \neq 0$, to obtain a nonnegative number $v_{n}(x)$, it is necessary that $b_{n} \leq 0$, this representing condition (2.9b).

Reciprocally, we assume that both (2.9a) and (2.9b) hold. These relations guarantee that $v_{n}(x)$ defined by (2.10) is a real nonnegative number for each $x \geq 0$ and $n \in \mathbb{N}$. Simple calculations show that $v_{n}(x)$ verifies $(2.8)$.

The proof is complete.

We proceed now to introduce the announced class of operators. Starting from (2.1), for each $n \in \mathbb{N}$, we define

$$
\left(L_{n}^{*} f\right)(x)=\sum_{k=0}^{\infty} u_{n, k}\left(v_{n}(x)\right) f\left(x_{n, k}\right), \quad x \geq 0, f \in \mathscr{F} \cap E_{\alpha},
$$

where $v_{n}$ is given by $(2.10)$.

The question now arises under which conditions this sequence is an approximation process.

Theorem 2.2. Let $L_{n}, n \in \mathbb{N}$, be defined by (2.1) such that (2.3), (2.4), and (2.9) hold. Let $L_{n}^{*}, n \in \mathbb{N}$, be defined by (2.11).

(i) The following identities

$$
L_{n}^{*} e_{0}=e_{0}, \quad L_{n}^{*} e_{1}=v_{n}, \quad L_{n}^{*} e_{2}=e_{2}
$$

hold.

(ii) One has $\lim _{n \rightarrow \infty} L_{n}^{*} f=f$ uniformly on compact subsets of $\mathbb{R}_{+}$for every $f \in \mathscr{F}_{+} \cap E_{\alpha}$. 


\section{Linear operators that preserve some test functions}

Proof. (i) Since (2.9) is fulfilled, $v_{n}, n \in \mathbb{N}$, are well defined. The first two identities are implied by $(2.3)$. Since $\left(L_{n}^{*} e_{2}\right)(x)=\left(L_{n} e_{2}\right)\left(v_{n}(x)\right)$, the last identity represents exactly relation (2.8).

(ii) The result follows from (2.12), Lemma 2.1, and Korovkin criterion.

Roughly speaking, Theorem 2.2 says: if $\left(L_{n}\right)_{n \geq 1}$ is an approximation process, then $\left(L_{n}^{*}\right)_{n \geq 1}$ inherits this property.

It would be interesting to present a new property of the sequence $\left(v_{n}\right)_{n \geq 1}$.

Lemma 2.3. Let $L_{n}, n \in \mathbb{N}$, be defined by (2.1) such that (2.3), (2.4), and (2.9) hold. For each $n \in \mathbb{N}$, the function $v_{n}$ given by (2.10) verifies

$$
v_{n}(0) \leq v_{n}(x) \leq x, \quad x \in \mathbb{R}_{+} .
$$

Proof. Examining (2.10), we deduce that $(d / d x) v_{n}(x) \geq 0, x \geq 0$, and this implies the first inequality. Combining relations (2.5) and (2.12), we obtain the second inequality.

\section{On the rate of convergence}

Here we explore the rate of convergence of $L_{n}^{*}, n \in \mathbb{N}$, operators in terms of both the modulus of continuity and of a certain weighted modulus. Based on the probabilistic interpretation of Korovkin's theorem, we will also give estimates in the frame of a probabilistic approach.

We denote by $C_{B}\left(\mathbb{R}_{+}\right)$the space of all bounded continuous real-valued functions on $\mathbb{R}_{+}$. Since $C_{B}\left(\mathbb{R}_{+}\right) \subset \mathscr{F} \cap E_{\alpha}$ and $\omega(f ; \cdot)$, the first modulus of smoothness of $f \in C_{B}\left(\mathbb{R}_{+}\right)$, is well defined, we state the following general estimate.

Theorem 3.1. Let $L_{n}, n \in \mathbb{N}$, be defined by (2.1) such that (2.3), (2.4), and (2.9) hold. Let $L_{n}^{*}, n \in \mathbb{N}$, be defined by (2.11). For each $n \in \mathbb{N}$, the operator $L_{n}^{*}$ verifies

(i) $\left\|L_{n}^{*}\right\|_{C_{B}\left(\mathbb{R}_{+}\right)}=1$,

(ii) for every $f \in C_{B}\left(\mathbb{R}_{+}\right)$,

$$
\left|\left(L_{n}^{*} f\right)(x)-f(x)\right| \leq\left(1+\frac{1}{\delta} \widetilde{v}_{n}(x)\right) \omega(f ; \delta), \quad x \geq 0, \delta>0
$$

(iii) for every $f$ differentiable on $\mathbb{R}_{+}$and $f^{\prime} \in C_{B}\left(\mathbb{R}_{+}\right)$,

$$
\left|\left(L_{n}^{*} f\right)(x)-f(x)\right| \leq\left|f^{\prime}(x)\right|\left(x-v_{n}(x)\right)+\widetilde{v}_{n}(x)\left(1+\frac{1}{\delta} \widetilde{v}_{n}(x)\right) \omega\left(f^{\prime} ; \delta\right)
$$

$x \geq 0, \delta>0$, where

$$
\tilde{v}_{n}(x):=\sqrt{2 x\left(x-v_{n}(x)\right)}, \quad x \geq 0,
$$

and $v_{n}$ is given by (2.10). 
Proof. Every operator $L_{n}^{*}$ maps continuously $C_{B}\left(\mathbb{R}_{+}\right)$into itself. Indeed, for $f \in C_{B}\left(\mathbb{R}_{+}\right)$ and $x \geq 0$, we have

$$
\left|\left(L_{n}^{*} x\right)(x)\right| \leq \sum_{k=0}^{\infty} u_{n, k}\left(v_{n}(x)\right)\|f\|_{\infty}=\|f\|_{\infty},
$$

consequently, $\left\|L_{n}^{*} f\right\|_{\infty} \leq\|f\|_{\infty}$. Here $\|\cdot\|_{\infty}$ stands for the sup-norm, $\|f\|_{\infty}:=\sup _{x \geq 0}$ $|f(x)|, f \in C_{B}\left(\mathbb{R}_{+}\right)$.

Moreover, $L_{n}^{*} e_{0}=e_{0}$, hence (i) holds.

For every $x \geq 0$, denote by $\psi_{x}: \mathbb{R}_{+} \rightarrow \mathbb{R}$ the function defined by $\psi_{x}(t)=t-x$. From (2.12) and (3.3), we get

$$
\begin{gathered}
\left(L_{n}^{*} \psi_{x}\right)(x)=v_{n}(x)-x, \\
\left(L_{n}^{*} \psi_{x}^{2}\right)(x)=\left(L_{n}^{*} e_{2}\right)(x)-2 x\left(L_{n}^{*} e_{1}\right)(x)+x^{2}\left(L_{n}^{*} e_{0}\right)(x)=\widetilde{v}_{n}^{2}(x) .
\end{gathered}
$$

For any positive linear operator $L$, see, for example, the monograph [1, Theorem 5.1.2], quantitative error estimates can be expressed in terms of the test functions as follows:

$$
|L f-f| \leq|f|\left|L e_{0}-1\right|+\left(L e_{0}+\frac{1}{\delta} \sqrt{\left(L e_{0}\right)\left(L \psi_{x}^{2}\right)}\right) \omega(f ; \delta),
$$

respectively,

$$
|L f-f| \leq|f|\left|L e_{0}-1\right|+\left|f^{\prime}\right|\left|L \psi_{x}\right|+\left(\sqrt{\left(L e_{0}\right)\left(L \psi_{x}^{2}\right)}+\frac{1}{\delta} L \psi_{x}^{2}\right) \omega\left(f^{\prime} ; \delta\right),
$$

$\delta>0$, provided $f \in C_{B}\left(\mathbb{R}_{+}\right)$, respectively, if $f$ is differentiable on $\mathbb{R}_{+}$and $f^{\prime} \in C_{B}\left(\mathbb{R}_{+}\right)$.

By virtue of these inequalities, taking in view both (2.12), (3.5) and (3.3), (2.13), the statements (ii) and (iii) follow.

The pointwise estimates allow us to obtain global estimates of the rate of convergence. For example, for every compact $K=[\mathrm{k}, \bar{k}] \subset \mathbb{R}_{+}$, (3.1) implies

$$
\left\|L_{n}^{*} f-f\right\|_{C(K)} \leq(1+\sqrt{2 \bar{k}}) \omega\left(f ; \sqrt{\bar{k}-v_{n}(\bar{k})}\right) .
$$

Indeed, if $x \in K$, then

$$
\tilde{v}_{n}(x) \leq \sqrt{2 \bar{k}}\left\{\max _{x \in K}\left(x-v_{n}(x)\right)\right\}^{1 / 2}=\sqrt{2 \bar{k}} \sqrt{\bar{k}-v_{n}(\bar{k})}
$$

and choosing in (3.1) $\delta:=\sqrt{\bar{k}-v_{n}(\bar{k})}$, we get the desired result. We may remark that even if a certain signal $f$ belongs to $C(K)$, we can extend it on $\mathbb{R}_{+}$taking $f(x)=f(\bar{k})$ for $x>\bar{k}$. By this way, $L_{n}^{*}$ is well defined. For the sake of simplicity, for the original signal and for its extension, we use the same notation. 
We proceed now to use again (3.6) for the initial operators $L_{n}, n \in \mathbb{N}$, obtaining

$$
\left|\left(L_{n} f\right)(x)-f(x)\right| \leq\left(1+\frac{1}{\delta} \sqrt{\left(a_{n}-1\right) x^{2}+b_{n} x+c_{n}}\right) \omega(f ; \delta),
$$

$x \geq 0, \delta>0$, where $f \in C_{B}\left(\mathbb{R}_{+}\right)$.

Another question can be asked: is the construction (2.11) useful from approximation theory point of view? Further on, we discuss the comparison between the upper bound of the errors generated by $L_{n}$ and that generated by $L_{n}^{*}$, respectively. Examining (3.10) and (3.1), we are concerned with finding $x \geq 0$ such that $L_{n}^{*} \psi_{x}^{2}(x) \leq L_{n} \psi_{x}^{2}(x)$ for each $n \in \mathbb{N}$. This means finding out the set

$$
M^{*}:=\left\{x \geq 0: x \sqrt{b_{n}^{2}+4 a_{n}\left(x^{2}-c_{n}\right)} \geq a_{n}\left(3-a_{n}\right) x^{2}+b_{n}\left(1-a_{n}\right) x-a_{n} c_{n}, n \in \mathbb{N}\right\} .
$$

Clearly, $M^{*} \neq \varnothing$ because of $0 \in M^{*}$, see (2.7). In the next section, in some particular cases, the explicit form of $M^{*}$ will be obtained.

Returning to Theorem 3.1, these results are not valid for any unbounded function $f$ because the classical modulus $\omega(f ; \cdot)$ is not defined. We can give estimates of the errors $\left|L_{n}^{*} f-f\right|, n \in \mathbb{N}$, involving unbounded functions by using a weighted modulus associated to the Banach space $\left(E_{\alpha},\|\cdot\|_{\alpha}^{*}\right), \alpha \geq 2$. In this respect, we consider

$$
\Omega_{w_{\alpha}}(f ; \delta):=\sup _{\substack{x \geq 0 \\ 0<h \leq \delta}} w_{\alpha}(x+h)|f(x+h)-f(x)|, \quad \delta>0,
$$

where the weight $w_{\alpha}$ is given by $w_{\alpha}(x)=\left(1+x^{\alpha}\right)^{-1}, x \geq 0$. It is evident that for each $f \in E_{\alpha}, \Omega_{w_{\alpha}}(f ; \cdot)$ is well defined and $\Omega_{w_{\alpha}}(f ; \delta) \leq 2\|f\|_{\alpha}^{*}, \delta>0, f \in E_{\alpha}$. We point out that in formula (3.12), the presence of the weight calculated at $x+h$ (not at $x$ ) is vital regarding some approximation properties of $\Omega_{w_{\alpha}}$, and this idea is due to Freud. In [5], weighted $L_{p}$-modulus of continuity with weights depending on two parameters has been considered.

Among some basic properties of $\Omega_{w_{\alpha}}(f ; \cdot)$ modulus, following [9], we state

$$
\begin{gathered}
\Omega_{w_{\alpha}}(f ; \lambda \delta) \leq(\lambda+1) \Omega_{w_{\alpha}}(f ; \delta), \quad \delta>0, \lambda>0, \\
\Omega_{w_{\alpha}}(f ; n \delta) \leq n \Omega_{w_{\alpha}}(f ; \delta), \quad \delta>0, n \in \mathbb{N}, \\
\lim _{\delta \rightarrow 0^{+}} \Omega_{w_{\alpha}}(f ; \delta)=0 .
\end{gathered}
$$

An accurate proof of the last property can be found in a recent paper of López-Moreno $[9$, Lemma 1].

Theorem 3.2. Let $L_{n}, n \in \mathbb{N}$, be defined by (2.1) such that (2.3), (2.4), and (2.9) hold. Let $L_{n}^{*}, n \in \mathbb{N}$, be defined by (2.11). For each $n \in \mathbb{N}$ and $f \in \mathscr{F}_{\mathcal{F}} \cap E_{\alpha}$, the operator $L_{n}^{*}$ verifies

$$
\left|\left(L_{n}^{*} f\right)(x)-f(x)\right| \leq \sqrt{\left(L_{n}^{*} \mu_{x}^{2}\right)(x)}\left(1+\frac{\tilde{v}_{n}(x)}{\delta}\right) \Omega_{w_{\alpha}}(f ; \delta), \quad x \geq 0, \delta>0,
$$


where $\tilde{v}_{n}$ is given at (3.3) and

$$
\mu_{x}(t):=1+(x+|t-x|)^{\alpha}, \quad t \geq 0 .
$$

Proof. Let $n \in \mathbb{N}$ and $f \in \mathscr{F} \cap E_{\alpha}$ be fixed. For each $t \in \mathbb{R}_{+}$and $\delta>0$, based on both definition (3.12) and on property (3.13) with $\lambda:=|t-x| \delta^{-1}$, we can write

$$
\begin{aligned}
|f(t)-f(x)| & \leq\left(1+(x+|t-x|)^{\alpha}\right)\left(|t-x| \delta^{-1}+1\right) \Omega_{w_{\alpha}}(f ; \delta) \\
& =\mu_{x}(t)\left(\delta^{-1}\left|\psi_{x}\right|(x)+1\right) \Omega_{w_{\alpha}}(f ; \delta) .
\end{aligned}
$$

Taking into account that $L_{n}^{*}$ is linear positive operator preserving the constants, we get

$$
\begin{aligned}
\left|\left(L_{n}^{*} f\right)(x)-f(x)\right| & =\left|L_{n}^{*}(f-f(x), x)\right| \leq L_{n}^{*}(|f-f(x)|, x) \\
& \leq L_{n}^{*}\left(\mu_{x}+\delta^{-1} \mu_{x}\left|\psi_{x}\right|, x\right) \Omega_{w_{\alpha}}(f ; \delta) .
\end{aligned}
$$

Cauchy-Schwarz inequality applied to $L_{n}^{*}$ gives

$$
L_{n}^{*} \mu_{x} \leq \sqrt{L_{n}^{*} \mu_{x}^{2}}, \quad L_{n}^{*} \mu_{x}\left|\psi_{x}\right| \leq \sqrt{L_{n}^{*} \mu_{x}^{2}} \sqrt{L_{n}^{*} \psi_{x}^{2}} .
$$

Since $\sqrt{L_{n}^{*} \psi_{x}^{2}}=\widetilde{v}_{n}$, the proof is finished.

To this end, we mention that the positive approximation processes can be studied by using probabilistic methods. This approach has its origin in the probabilistic interpretation of Korovkin's theorem due to King [7]. At the moment, our aim is to present the significance of relation (2.12) from this point of view.

In a probability space $(\Omega, \mathcal{K}, P)$, we consider a random scheme $Z$ on $\mathbb{R}_{+}$, for a thorough documentation, [1, Section 5.2] can be consulted. The random variables $Z(n, x),(n, x) \in$ $\mathbb{N} \times \mathbb{R}_{+}$, are discretely distributed such that the distribution $P_{Z(n, x)}$ of $Z(n, x)$ is given by $P_{Z(n, x)}=\sum_{k=0}^{\infty} u_{n, k}\left(v_{n}(x)\right) \varepsilon_{x_{n, k}}$, where $\varepsilon_{x_{n, k}}$ denotes the unit mass at $x_{n, k}$. For every $f \in$ $C_{B}\left(\mathbb{R}_{+}\right)$, we can consider the operators defined by $(2.11)$. Let $E(Z(n, x))$, $\operatorname{Var}(Z(n, x)):=$ $\sigma_{n, x}^{2}$ be the expected value and the variance of $Z(n, x)$, respectively. Since (2.12) holds, we get

$$
E(Z(n, x))=v_{n}(x), \quad \operatorname{Var}(Z(n, x))=E\left(Z^{2}(n, x)\right)-E^{2}(Z(n, x))=x^{2}-v_{n}^{2}(x) .
$$

Consequently, in broad outlines,

$$
x^{2}=\sigma_{n, x}^{2}+v_{n}^{2}(x), \quad x \geq 0, n \in \mathbb{N},
$$

represents the characteristic relation generated by our operators $L_{n}^{*}, n \in \mathbb{N}$, in a probabilistic frame.

\section{Examples}

Starting from some known approximation processes verifying conditions (2.1)-(2.4), (2.9), with Section 2 in mind, we focus our attention on obtaining modified processes 
of $L_{n}^{*}$-type. The linear positive operators we are referring to are the following: SzászMirakjan-Favard, Baskakov, Bernstein-Chlodvsky operators, all of them being unitary presented, for example, in the monograph of Altomare and Campiti [1, Chapter 5]. We also mention that the first two classes have the same equidistant net $\Delta_{n}=(k / n)_{k \geq 0}$.

(1) Szász-Mirakjan-Favard operators. For this class, the identities $a_{n}=1, b_{n}=1 / n$, $c_{n}=0, n \in \mathbb{N}$, hold, consequently conditions (2.4) and (2.9) are fulfilled. Thus

$$
v_{n}(x)=\frac{\sqrt{1+4 n^{2} x^{2}}-1}{2 n}, \quad x \geq 0, n \in \mathbb{N},
$$

and the corresponding modified operators are defined by

$$
\left(L_{n}^{*} f\right)(x):=e^{-n v_{n}(x)} \sum_{k=0}^{\infty} \frac{\left(n v_{n}(x)\right)^{k}}{k !} f\left(\frac{k}{n}\right), \quad x \geq 0, n \in \mathbb{N},
$$

their domain can be taken $E_{2}$.

A short computation gives $M^{*}=[0, \infty)$, where $M^{*}$ was introduced by (3.11).

(2) Baskakov operators. In this case, relation (2.4) is verified by the sequences $a_{n}=$ $1+1 / n, b_{n}=1 / n, c_{n}=0, n \in \mathbb{N}$. We get

$$
v_{n}(x)=\frac{\sqrt{1+4 n(n+1) x^{2}}-1}{2(n+1)}, \quad x \geq 0, n \in \mathbb{N}
$$

and, following (2.11), the modified Baskakov operators are defined by

$$
\left(L_{n}^{*} f\right)(x):=\sum_{k=0}^{\infty}\left(\begin{array}{c}
n+k-1 \\
k
\end{array}\right) \frac{v_{n}^{k}(x)}{\left(1+v_{n}(x)\right)^{n+k}} f\left(\frac{k}{n}\right), \quad x \geq 0, n \in \mathbb{N},
$$

where $f \in E_{2}$. With regard to the set $M^{*}$, by a straightforward calculus, we obtain $M^{*}=$ $[0, \infty)$.

We notice that $M^{*}$ associated to each of the above two examples guarantee that the order of approximation of $\left(L_{n}^{*} f\right)(x)$ to $f(x)$ is at least as good as the order of approximation to $f(x)$ by $\left(L_{n} f\right)(x)$, for any $x \in \mathbb{R}_{+}$.

(3) Bernstein-Chlodovsky operators. Let $\left(h_{n}\right)_{n \geq 1}$ be a sequence of strictly positive real numbers verifying $\lim _{n \rightarrow \infty} h_{n}=\infty$ and

$$
\lim _{n \rightarrow \infty} \frac{h_{n}}{n}=0
$$

The $n$th Bernstein-Chlodovsky operator, $L_{n}: C\left(\mathbb{R}_{+}\right) \rightarrow C\left(\mathbb{R}_{+}\right), n \in \mathbb{N}$, is defined by

$$
\left(L_{n} f\right)(x):= \begin{cases}\sum_{k=0}^{n}\left(\begin{array}{l}
n \\
k
\end{array}\right)\left(\frac{x}{h_{n}}\right)^{k}\left(1-\frac{x}{h_{n}}\right)^{n-k} f\left(\frac{h_{n} k}{n}\right), & \text { if } 0 \leq x \leq h_{n}, \\
f(x), & \text { if } x>h_{n} .\end{cases}
$$


It is known that the identity (4.5) ensures $\lim _{n \rightarrow \infty} L_{n} f=f$ in $E_{2}$, and hence uniformly on compact subsets of $\mathbb{R}_{+}$. This time, we obtain

$$
\begin{gathered}
a_{n}=1-\frac{1}{n}, \quad b_{n}=\frac{h_{n}}{n}, \quad c_{n}=0, \quad \text { if } x \in\left[0, h_{n}\right], \\
a_{n}=1, \quad b_{n}=c_{n}=0, \quad \text { if } x>h_{n} .
\end{gathered}
$$

Taking in view the requirements of Section 2, at first cast of glance, something seems wrong because (2.7) does not hold for $n=1\left(a_{1}=0\right)$. The reason is simple: since now relation (2.6) must hold only for $x \in\left[0, h_{n}\right]$, not for $x \in \mathbb{R}_{+}$, condition $a_{n} \geq 1$ in (2.7) is not necessary to take place. It is not difficult to find the sequence $\left(v_{n}\right)_{n \geq 1}$. We get $v_{1}(x)=x^{2}, x \geq 0$. For $n \geq 2$,

$$
v_{n}(x)= \begin{cases}\frac{1}{2(n-1)}\left(\sqrt{h_{n}^{2}+4 n(n-1) x^{2}}-h_{n}\right), & \text { if } x \in\left[0, h_{n}\right], \\ x, & \text { if } x>h_{n} .\end{cases}
$$

Returning to (4.6) via (2.11), we obtain $L_{n}^{*}$.

In order to indicate $M^{*}$, we infer that for each $n \geq 2$, the inequality incorporated in (3.11) is verified by those $x$ belonging to the set $M_{n}^{*}=\left[0,((n+1) /(3 n+1)) h_{n}\right] \cup\left[h_{n}, \infty\right)$. Also, $M_{1}^{*}=\mathbb{R}_{+}$. We get

$$
M^{*}=\bigcap_{n \geq 1} M_{n}^{*}=\bigcap_{n \geq 2}\left[0, \frac{n+1}{3 n+1} h_{n}\right] .
$$

Putting $h^{*}:=\min _{n \geq 1} h_{n}$, clearly $\left[0, h^{*} / 3\right] \subset M^{*}$, consequently the order of approximation of $L_{n}^{*} f$ to $f$ is at least as good as the order of approximation of $L_{n} f$ to $f$ whenever $0 \leq x \leq h^{*} / 3$.

If we choose in (4.6) $h_{n}=1$, then, for $x \in[0,1],\left(L_{n} f\right)(x)$ becomes the classical $n$th Bernstein polynomial for each $n \in \mathbb{N}$. Our sequence $\left(v_{n}\right)_{n \geq 1}$ is identical to the result of King [8, Equation (2.2)].

\section{Approaches in abstract spaces}

A fruitful research direction has consisted in extensions of Bohman-Korovkin theorem to abstract spaces and, as a result, the so-called KAT, meaning Korovkin-type Approximation theory, has been developed. Motivated by these researches, in the present section, we deal with establishing what the condition $L_{n}^{*} e_{2}=e_{2}$ would become in certain abstract spaces, such as Hilbert spaces. In this direction, the book of Debnath and Mikusinski [4] is a complete and accessible text containing all important information about the mentioned spaces.

Case 1. Let $E$ be a real vector space with an inner product $(\cdot, \cdot)$. Let $X \subset E$ be a compact set. $C(X, \mathbb{R})$ stands for the Banach lattice of all real-valued continuous functions on $X$, endowed with the sup-norm $\|\cdot\|$. We consider the functions $1_{X}, e, e_{x}: X \rightarrow \mathbb{R}, x \in X$, defined as follows:

$$
1_{X}(t):=1, \quad e(t):=(t, t), \quad e_{x}(t):=(x, t), \quad t \in X .
$$


Our concern is to select which of these functions could play the role of the classical monomial $e_{2}$. If we take the particular case $E:=\mathbb{R}, X:=[a, b]$, and the inner product is the usual multiplication, then $e(t)=t^{2}=e_{2}(t)$ and $e_{x}(t)=x e_{1}(t), t \in[a, b]$. With this fact in mind, based on the results established by Andrica and Mustăţa [2], we can state the following theorem.

THeOREm 5.1. Let $\left(L_{n}\right)_{n \geq 1}$ be a sequence of linear positive operators acting on $C(X, \mathbb{R})$ such that $L_{n} 1_{X}=1_{X}$ and $L_{n} e=e, n \in \mathbb{N}$, hold.

(i) If $f \in \operatorname{Lip}(X, \mathbb{R})$, then $\left\|L_{n} f-f\right\| \leq \sqrt{2} c_{f}\left\|L_{n} e_{x}-e\right\|^{1 / 2}, x \in X$.

(ii) If $\left(\left(L_{n} e_{x}\right)(x)\right)_{n \geq 1}$ converges to $e(x)$ uniformly for $x \in X$, then $\left(L_{n} f\right)_{n \geq 1}$ converges uniformly to $f$ for all $f \in C(X, \mathbb{R})$.

Proof. For any linear positive operator $L_{n}$ acting on $C(X, \mathbb{R})$ and verifying $L_{n} 1_{X}=1_{X}$ (this means a Markov operator), one has

$$
\left\|L_{n} f-f\right\| \leq c_{f}\left\|a_{n}-2 b_{n}\right\|^{1 / 2}, \quad \text { for each } f \in \operatorname{Lip}(X, \mathbb{R})
$$

where $a_{n}(x):=\left(L_{n} e\right)(x)-e(x)$ and $b_{n}(x):=\left(L_{n} e_{x}\right)(x)-e(x), x \in X, n \in \mathbb{N}$, see [2, Equation (6) ]. In our case, $a_{n}=0$ and the first statement is proved.

Following [2, Theorem 4] closely, under our assumptions, we can write

$$
L_{n}((\cdot-x, \cdot-x), x)=2\left(e(x)-\left(L_{n} e_{x}\right)(x)\right), \quad n \in \mathbb{N} \text {. }
$$

Since the subspace $\operatorname{Lip}(X, \mathbb{R})$ is dense in $C(X, \mathbb{R})$ with respect to the uniform norm, the statement (i) implies the statement (ii).

Case 2. Let $E$ be a real Hilbert space. We denote by $\|\cdot\|_{E}$ the norm induced by its inner product $(\cdot, \cdot),\|x\|_{E}=\sqrt{(x, x)}, x \in E$. Let $X \subset E$ be a compact convex set. Letting

$$
\begin{gathered}
D_{X}^{1}(E):=\{f: E \longrightarrow \mathbb{R}: f \text { is Gâteaux derivable on } X, \\
\left.f^{\prime}: X \longrightarrow E^{*}, f^{\prime}(x)=f_{x}^{\prime}, \text { is continuous on } X\right\}, \\
C^{1}(X):=\left\{\left.f\right|_{X}: f \in D_{X}^{1}(E)\right\},
\end{gathered}
$$

we recall $f: E \rightarrow \mathbb{R}$ is Gâteaux derivable at $x \in X$ if, for all $h \in E$, there exists $\lim _{t \rightarrow 0}(f(x+$ $t h)-f(x)) / t:=f_{x}^{\prime}(h)$ and $f_{x}^{\prime}$ is linear and continuous as function in $h$, that is, $f_{x}^{\prime} \in E^{*}$. The function $f$ is Gâteaux derivable on $X$ if $f_{x}^{\prime} \in E^{*}$ for each $x \in X$. Here $E^{*}$ is endowed with the usual norm $\|\cdot\|_{E^{*}}$,

$$
\left\|x^{*}\right\|_{E^{*}}:=\sup \left\{\left|x^{*}(x)\right|: x \in E,\|x\|_{E} \leq 1\right\}, \quad x^{*} \in E^{*} .
$$

For the sake of completeness, we also recall the modulus of continuity of a function $g$ : $E \rightarrow F$ on $X,\left(F,\|\cdot\|_{F}\right)$ is real normed space, is given by

$$
\omega(g ; \delta):=\sup \left\{\|g(x)-g(y)\|_{F}: x, y \in X,\|x-y\|_{E} \leq \delta\right\}
$$

$\delta \in[0, \rho(X)]$, where $\rho(X)$ represents the diameter of the compact set $X$. 
Theorem 5.2. Let $L: C(X, \mathbb{R}) \rightarrow C(X, \mathbb{R})$ be a linear positive operator satisfying the properties $L 1_{X}=1_{X}$ and $L e=e$. For each $f \in C^{1}(X)$ and each $x \in X$ such that $(L f)(x) \neq f(x)$, one has

$$
|(L f)(x)-f(x)| \leq\left|L\left(f_{x}^{\prime}(\cdot-x), x\right)\right|+4 \Psi_{L}(x) \omega\left(f^{\prime} ; \frac{\left(e-L e_{x}\right)(x)}{\Psi_{L}(x)}\right),
$$

where $\Psi_{L}(x)=L\left(\|\cdot-x\|_{E}, x\right)$ and the functions $1_{X}, e, e_{x}$ are given by (5.1).

The requirement $(L f)(x) \neq f(x)$ guarantees that $\Psi_{L}(x)>0$. This theorem has an origin the work of $\mathrm{Gal}$ representing a slight modification of his result [6, Theorem 3.1] under our additional hypothesis $L e=e$. So, we omit the proof.

\section{Acknowledgment}

The work is supported by Grant CNCSIS 355/2005-2006.

\section{References}

[1] F. Altomare and M. Campiti, Korovkin-Type Approximation Theory and Its Applications, de Gruyter Studies in Mathematics, vol. 17, Walter de Gruyter, Berlin, 1994.

[2] D. Andrica and C. Mustăţa, An abstract Korovkin type theorem and applications, Studia Universitatis Babeş-Bolyai. Mathematica 34 (1989), no. 2, 44-51.

[3] G. Bleimann, P. L. Butzer, and L. Hahn, A Bernstein-type operator approximating continuous functions on the semi-axis, Indagationes Mathematicae 42 (1980), no. 3, 255-262.

[4] L. Debnath and P. Mikusinski, Introduction to Hilbert Spaces with Applications, 3rd ed., Elsevier, Massachusetts, 2005.

[5] G. Freud, Investigations on weighted approximation by polynomials, Studia Scientiarum Mathematicarum Hungarica 8 (1973), 285-305.

[6] S. G. Gal, Approximation of continuously Gâteaux derivable functionals by Markov operators, Revue d'Analyse Numérique et de Théorie de l'Approximation 25 (1996), no. 1-2, 111-119.

[7] J. P. King, Probabilistic analysis of Korovkin's theorem, The Journal of the Indian Mathematical Society. New Series 44 (1980), no. 1-4, 51-58 (1982).

[8] _ Positive linear operators which preserve $x^{2}$, Acta Mathematica Hungarica 99 (2003), no. 3, 203-208.

[9] A.-J. López-Moreno, Weighted simultaneous approximation with Baskakov type operators, Acta Mathematica Hungarica 104 (2004), no. 1-2, 143-151.

Octavian Agratini: Faculty of Mathematics and Computer Science, Babeş-Bolyai University, 400084 Cluj-Napoca, Romania

E-mail address: agratini@math.ubbcluj.ro 


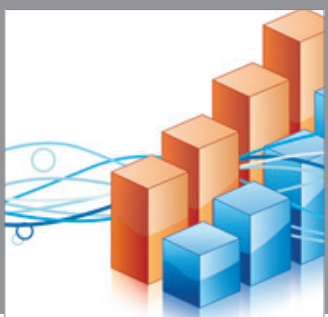

Advances in

Operations Research

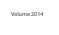

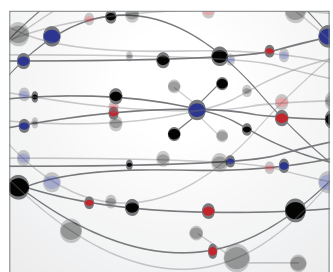

\section{The Scientific} World Journal
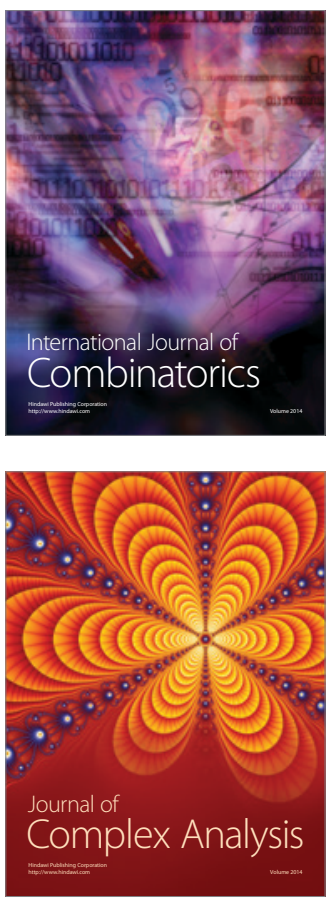

International Journal of

Mathematics and

Mathematical

Sciences
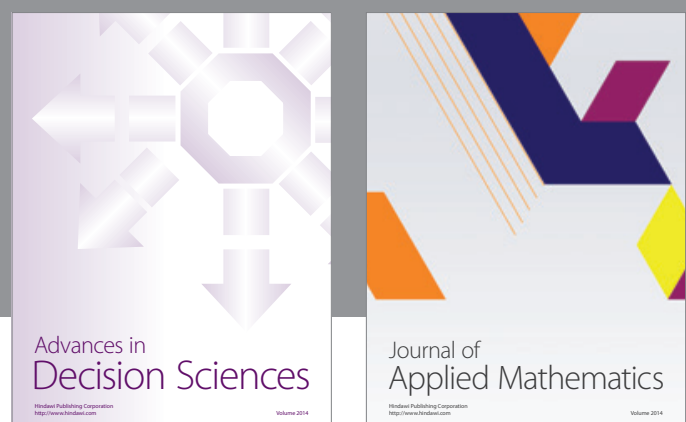

Journal of

Applied Mathematics
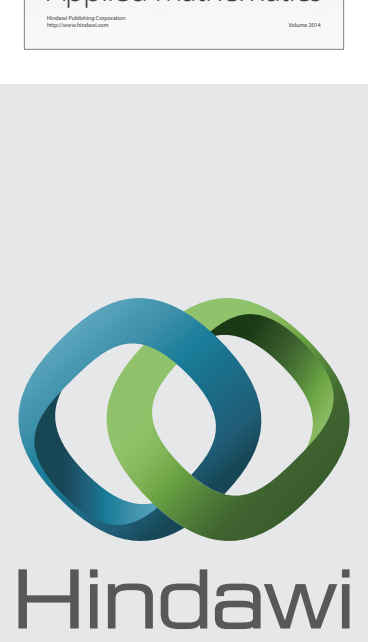

Submit your manuscripts at http://www.hindawi.com
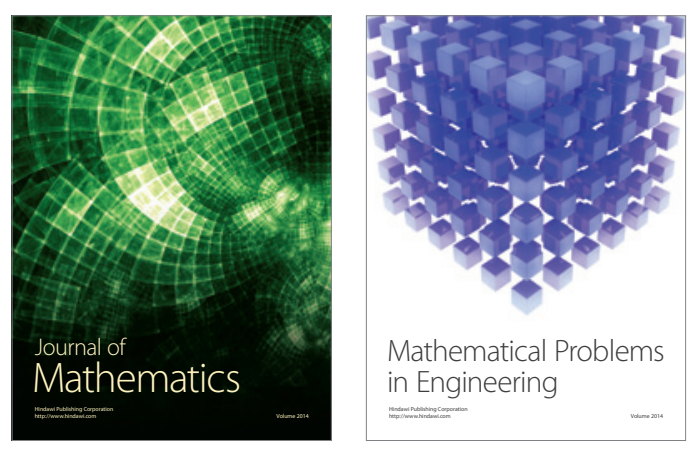

Mathematical Problems in Engineering
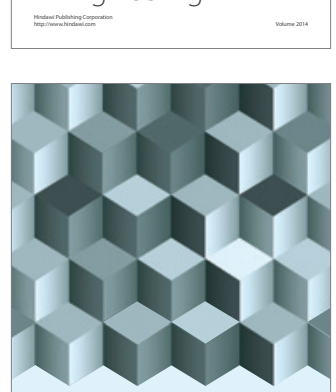

Journal of

Function Spaces
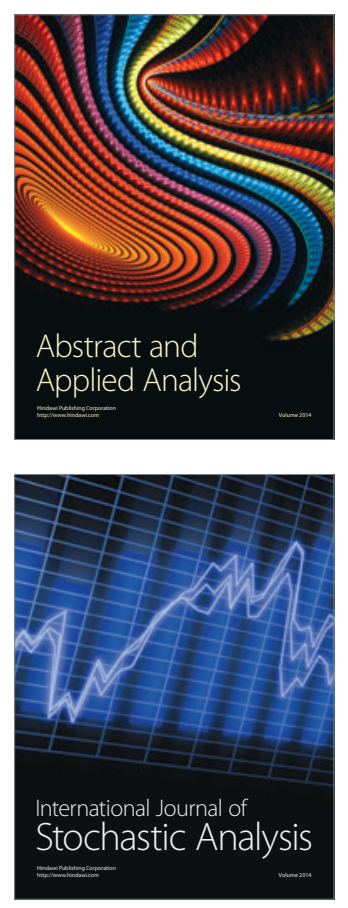

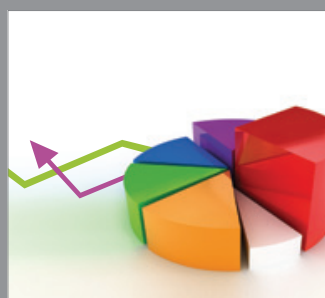

ournal of

Probability and Statistics

Promensencen
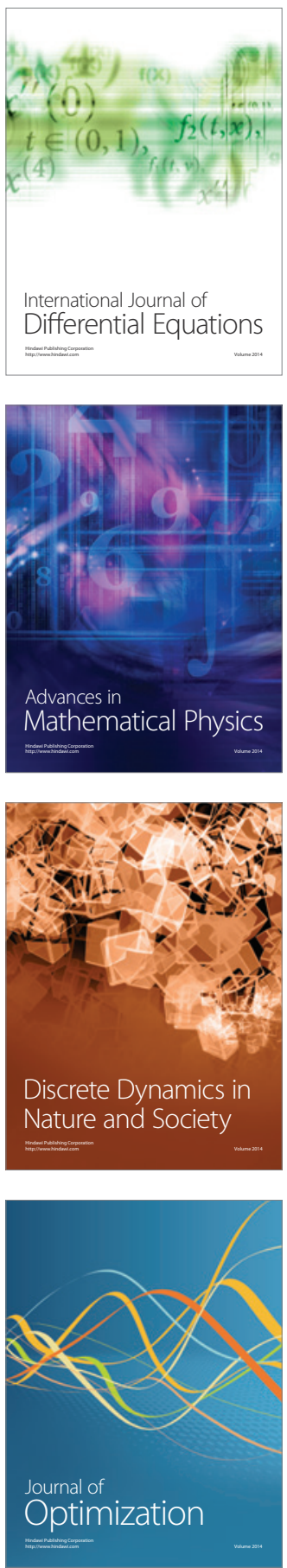